\title{
THERMAL EXPANSION OF GaN BULK CRYSTALS AND HOMOEPITAXIAL LAYERS
}

\author{
M. Leszczyński, H. Teisseyre, T. Suski, I. Grzegory, M. Boćkowski, \\ J. Jun, B. Palosz, S. Porowski
}

High Pressure Research Center, Polish Academy of Sciences

Sokołowska 29/37, 01-142 Warsaw, Poland

K. Pakula, J.M. Baranowski

Institute of Experimental Physics, Warsaw University, Hoża 69, 00-681 Warsaw, Poland

\author{
AND A. BARSKI
}

CEA-Grenoble, Departement de Recherche Fondamentale sur la Matiere Condensée SP2M, 17 Rue des Martyrs, 38054 Grenoble Cedex 9, France

Thermal expansion of gallium nitride was measured using high resolution X-ray diffraction. The following samples were examined: (i) single monocrystals grown at pressure of about $15 \mathrm{kbar}$, (ii) homoepitaxial layers. The main factor influencing both, the lattice parameters and the thermal expansion coefficient, are free electrons related to the nitrogen vacancies. The origin of an increase in the lattice constants by free electrons is discussed in terms of the deformation potential of the conduction-band minimum. An increase of the thermal expansion by free electrons is explained by a decrease of elastic constants.

PACS numbers: $61.72 . J i, 61.72 . \mathrm{Vv}$

\section{Introduction}

The lattice parameters of semiconductors depend on the following factors: (i) concentrations of dopants or native defects which contract or expand the lattice due to the size effect, (ii) concentration of free charge which changes the lattice parameters via the deformation potential of the extremum occupied by this charge [1-5], (iii) internal and external stresses (for example, due to the presence of a mismatched substrate), (iv) temperature. The latter factor may induce the change of lattice parameters not only by anharmonicity of thermal vibrations, but also by a redistribution of the free charge in the conduction- or the valence-band. The four above-mentioned factors are not independent from each other. The relation between free charge and dopant concentrations is usually a straightforward one. 
More complicated relations characterize other factors. Recently, it has been pointed out (Ref. [6]) that doping (free charge) influences anharmonicity of the thermal vibrations.

In our recent papers [7-9] the experience gained from the studies on $\mathrm{Si}$ [1], GaAs $[2,3]$ and AlGaAs [4-6] was applied in the explanation of some features of the microstructure of GaN single crystals and epitaxial layers. This material attracts a considerable interest because of the present and future applications in blue-range optoelectronics and high-temperature electronics. However, gallium nitride is extremely difficult to grow. This can be done at high hydrostatic pressures of about $15 \mathrm{kbar}$ and temperatures of about $1800 \mathrm{~K}$ [10]. In this method, the crystals are self-seeded on the surface of the gallium droplet placed in the pressure chamber with nitrogen as a pressure transmitting medium. Then, the crystals grow as hexagonal plates (wurtzite structure, (0001)-oriented) into the gallium inside. In such growth conditions the crystals are gallium-rich, i.e., with the high concentration of nitrogen vacancies (responsible [11] for the free-electron concentration of about $5 \times 10^{19} \mathrm{~cm}^{-3}$ ) and gallium antisites. All three factors (N-vacancies, free electrons, Ga-antisites) expand the lattice. In our previous paper [9] we discussed this problem pointing out that the main contribution to the lattice-parameter increase is due to free electrons via the deformation potential of the conduction band minimum of about $-10 \mathrm{eV}$ [12]. However, it was not possible to perform a quantitative analysis of all factors influencing the lattice parameters of $\mathrm{GaN}$ because some bits of necessary information were not available. In particular, the concentration of gallium antisites was unknown, neither the change of the thermal expansion caused by the presence of free electrons released by nitrogen vacancies.

The aim of this paper is to give an answer to the latter point. For our investigations we used the following samples: (i) bulk crystals of rough sides, (ii) bulk crystals of smooth sides, (iii) homoepitaxial layers grown by metalorganic chemical vapour deposition (MOCVD). The difference between two sides of the bulk crystals is induced by the precipitation of gallium atoms near the rough sides, resulting in the decrease in the lattice parameters [8]. The main difference between the bulk crystals and the homoepitaxial layers, is a much lower (about $10^{17} \mathrm{~cm}^{-3}$ ) concentration of the free electrons in the layers.

\section{Experimental}

The measurements were performed at temperatures $40-770 \mathrm{~K}$ for the bulk crystals and $77-770 \mathrm{~K}$ for the homoepitaxial layers. The lattice parameters were measured either by using the Bond method [13] or by a direct measurement of the Bragg angle with the channel-cut analyser in the Philips MRD diffractometer or $10 \mathrm{~mm}$ slit placed at a distance of about $700 \mathrm{~mm}$ of the diffractometer of ID-32 beam-line of the European Synchrotron Radiation Facility (ESRF) in Grenoble. The reflections 00.4 and $00.6 \mathrm{Cu} K_{\alpha_{1}}$ served for evaluating the $c$-parameters (perpendicular to the surface); $22.0,11.3$ and 11.4 for evaluating $a$-parameters.

\section{Results and discussion}

The lattice parameters of the rough- and smooth-side of the bulk crystal, as well as of the homoepitaxial layer grown on the rough side of the bulk crystal, are 
given in Table. It can be seen that the difference between the lattice parameters of these three kinds of samples increases at high temperatures. This means that the thermal expansion is larger for samples whose lattice is expanded (most probably by the free electrons).

TABLE

Lattice parameters of gallium nitride bulk crystals and homoepitaxial (MOCVD-grown) layers. Lattice parameters $a$ for the homoepitaxial layer were the same as for the bulk (rough side) crystal.

\begin{tabular}{r|c|c|c|c|c}
\hline \hline \multirow{2}{*}{$T[\mathrm{~K}]$} & \multicolumn{2}{|c|}{$a[\AA]$} & \multicolumn{3}{c}{$c[\AA]$} \\
\cline { 2 - 6 } & bulk & bulk & bulk & bulk & homo-epitax. \\
rough-side & smooth-side & rough-side & smooth-side & \\
\hline 40 & 3.1866 & 3.1873 & 5.1836 & 5.1842 & not measured \\
77 & 3.1868 & 3.1876 & 5.1838 & 5.1844 & 5.1828 \\
150 & 3.1872 & 3.1880 & 5.1844 & 5.1850 & 5.1834 \\
200 & 3.1875 & 3.1885 & 5.1848 & 5.1856 & 5.1837 \\
295 & 3.1880 & 3.1890 & 5.1856 & 5.1864 & 5.1844 \\
400 & 3.1891 & 3.1901 & 5.1870 & 5.1878 & 5.1857 \\
500 & 3.1906 & 3.1917 & 5.1886 & 5.1895 & 5.1872 \\
600 & 3.1920 & 3.1932 & 5.1908 & 5.1918 & 5.1893 \\
700 & 3.1935 & 3.1948 & 5.1936 & 5.1946 & 5.1923 \\
770 & 3.1950 & 3.1964 & 5.1958 & 5.1970 & 5.1945 \\
\hline \multicolumn{2}{|c|}{ \pm 0.0004} & \multicolumn{3}{c}{ \pm 0.0001} \\
\end{tabular}

This finding is similar to the one observed for AlGaAs:Te [6] for which it was proposed that free electrons soften the lattice as it is so for germanium or silicon [14]. However, the change of thermal expansion versus free electrons for $\mathrm{GaN}$ is smaller than for AlGaAs, which can be understood taking into account a much higher hardness of GaN.

The most probable factor inducing the change of the thermal expansion are free electrons by themselves. However, in the bulk crystals of gallium nitride the presence of free electrons is related to the vacancies and to clarify this problem further research is needed on samples in which dopants are the source of free electrons.

The main result of the presented work is the finding that differences in thermal expansion are a secondary-importance factor influencing the lattice constants of GaN. The next task leading to the understanding of the lattice constant changes in $\mathrm{GaN}$ will be to estimate the concentration of gallium antisites and how nitrogen vacancies expand the lattice. Knowing these it will be possible to find an experimental value of the deformation potential of the conduction band minimum for gallium nitride. 


\section{Acknowledgments}

The work was sponsored by the Committee for Scientific Research, grant no. 175/PO3/96/10. The authors are indebted to Dr F. Rieutord of the D32 ESRF beam line for his help in the experiments.

\section{References}

[1] G.S. Cargill III, J. Angillelo, K.J. Kavanagh, Phys. Rev. Lett. 61, 1748 (1988).

[2] J. Bąk-Misiuk, H.G. Brühl, W. Paszkowicz, U. Pietsch, Phys. Status Solidi A 106, 451 (1988).

[3] M. Leszczyński, J. Bąk-Misiuk, J. Domagała, J. Muszalski, M. Kaniewska, J. Marczewski, Appl. Phys. Lett. 67, 539 (1995).

[4] G.S. Cargill III, A. Segmüller, T.F. Kuech, T.N. Theis, Phys. Rev. B 46, 10078 (1992).

[5] M. Leszczyński, J. Bąk-Misiuk, J. Domagała, Acta Phys. Pol. A 88, 837 (1995).

[6] J. Bakk-Misiuk, M. Leszczyński, J. Domagała, Z. Żytkiewicz, J. Appl. Phys., in press.

[7] M. Leszczyński, T. Suski, P. Perlin, H. Teisseyre, I. Grzegory, M. Boćkowski, J. Jun, S. Porowski, J. Major, J. Phys. D, Appl. Phys. 28, 1 (1995).

[8] M. Leszczyński, I. Grzegory, G. Teisseyre, T. Suski, M. Boćkowski, J. Jun, S. Porowski, J. Cryst. Growth, in press.

[9] M. Leszczyński, T. Suski, H. Teisseyre, I. Grzegory, M. Boćkowski, J. Jun, K. Pakuła, J.M. Baranowski, S. Porowski, T.C. Foxon, Appl. Phys. Lett., in press.

[10] S. Porowski, I. Grzegory, J. Jun, in: High Pressure Chemical Synthesis, Eds. J. Jurczak, B. Baranowski, Elsevier, Amsterdam 1989, p. 21.

[11] P. Perlin, T. Suski, H. Teisseyre, M. Leszczyński, I. Grzegory, J. Jun, S. Porowski, P. Bogusawski, J. Bernholc, J.V. Chervin, A. Polian, T.D. Moustakas, Phys. Rev. Lett. 75, 296 (1995).

[12] P. Perlin, I. Gorczyca, N.E. Christensen, I. Grzegory, H. Teisseyre, T. Suski, Phys. Rev. B 45, 13307 (1992).

[13] W.L. Bond, Acta Crystallogr. 13, 814 (1960).

[14] V.V. Zhidanova, T.A. Kontorova, Sov. Phys.-Solid State 7, 2685 (1966). 